\title{
Performance Analysis of Asynchronous Carrier Interferometry/MC-CDMA Uplink with Interference Cancellation Techniques
}

\author{
Vijaya Thippavajjula \\ Balasubramaniam Natarajan \\ Department of Electrical and Computer Engineering \\ Kansas State University. Manhattan. KS, 66506 \\ Phone: (+l) 785532 5600. Fax: (+1) 7855321188 \\ E-mail: $\{$ vijayat.bala $\} @ k s u, e d u$
}

\begin{abstract}
In this paper, we analyze the uplink performance of an asynchronous Carrier Interferometry/MC-CDMA, with nonlinear parallel and serial interference cancellation techniques. Specifically, we employ the new variant of Parallel Interference Cancellation known as Block-PIC and demonstrate significant performance (10 dB) improvement relative to conventional PIC. We also study the effect of order of cancelling in serial interference cancellation by comparing the performance of the conventional fixed ordering scheme with dynamic ordering. We show that dynamic ordering provides a gain of $8 \mathrm{~dB}$ relative to fixed ordering.
\end{abstract}

Keywords: Parallel Interference Cancellation, Serial Interference Cancellation, Frequency-Selective Fading, MC-CDMA, Carrier Interferometry (CI).

\section{INTRODUCTION}

The demand for high data rate transmissions has motivated the selection of MC-CDMA as a candidate multiple access technique for $4 G$ cellular systems. MC-CDMA is characterized by high spectral efficiency. large system capacity and high flexibility in data rate. In MC-CDMA. each user's data symbol is transmitted simultaneously over $N$ narrow band carriers. with each subcarrier encoded with an element of the spreading code. Users are assigned unique, orthogonal (or pseudo-orthogonal) codes. Recently a new spreading code set called Carrier Interferometry (CI) was employed in MC-CDMA system and was shown to improve the performance as well as the capacity [1].

The CI/MC-CDMA downlink performance analysis has been presented in [1]. However there is little to no work on the performance of CV/MC-CDMA uplink. In uplink transmission, the base station receives the signals from different mobile terminals through different paths and every user's signal experiences independent random amplitude and phase distortions resulting in the loss of orthogonality at the receiver. This in turn gives rise to multiple access interference (MAI) which limits the capacity and performance of the system. Multiuser detection is a powerful technique to combat MAI. The optimum multiuser detector proposed in [2] achieves significant performance improvement relative to single-user receivers but the computational complexity increases exponentially with the number of users. This has motivated the use of low-complexity linear [3] and nonlinear decision-driven suboptimal multituser detection techniques.

Nonlinear, decision-driven multiuser detection techniques can be divided into two broad categories - successive and parallel interference cancellation. In successive interference cancellation (SIC), interference due to other users is cancelled sequentially [4]. This process involves a large decoding delay to accomplish interference cancellation for all the users. Pipelined SIC (pSIC) scheme has been proposed [5] to compensate the problem of decoding delay and is shown to have a better performance than SIC with same decoding delay. Further improvement in the performance is achieved by adaptive pSIC [6]. In [7], we studied the effect of order of cancelling users on the BER performance for synchronous systems and proposed new dynamic ordering schemes. A significant performance improvement is achieved by dynamic ordering with order being updated after each cancellation.

In case of parallel interference cancellation (PIC), the MAI experienced by the desired user is cancelled in one shot. PIC requires more hardware than SIC but is more attractive due to its higher speed. The performance of these interference cancellation techiniques depends orl the precise estimation of MAI. To improve the reliability of interference estimation, a different method called partial PIC (pPIC) is discussed in [8] and is shown to have a better performance than conventional PIC. In case of $\mathrm{pPIC}$. the interference is cancelled partially depending on the reliability of the estimated data. In [9], we proposed two variants of PIC known as threshold-PIC(TPIC) and Block-PIC. Block-PIC is shown to provide the best performance in synchronous systems.

In this work, we characterize asynchronous CI/MC-CDMA uplink performance and is the first attempt in doing so, to the best of authors knowledge. We evaluate the effectiveness of using conventional methods of PIC and SIC. Next we employ the new variants of non linear detection techniques, that were proposed for synchronous systems. Block-PIC: In this method, all users are divided into Block 1 (strong users) and Block 2 (weak users) based on the magnitude of their received signals. PIC is first performed within Block 1 and using the estimated data the interference due to Block $\mathbf{I}$ is removed from Block 2. PIC is then employed within Block 2 and the weak user's data is estimated. It can be observed from the performance results that, the Block-PIC significantly outperforms conventional PIC providing $10 \mathrm{~dB}$ gain at a BER of $10^{-2}$.

In conventional SIC, the ordering followed is fixed. That is, users are ordered based on their signal strengths and this order is kept fixed through out the interference cancellation process. In this work we study the effect of dynamic ordering (DO) where, users are initially ordered based on their signal strengths and this order is updated after each cancellation. We compare the performance of SIC receivers with fixed ordering scheme and dynamic ordering, updating after each cancellation. We demonstrate that SIC employing dynamic ordering provides a $8 \mathrm{~dB}$ gain relative to SIC employing fixed ordering at BER of $2 \cdot 10^{-3}$.

This paper is organized as follows: Section II outlines the system model describing the transmitter, channel model and the receiver structure. Section III describes the Block-PIC technique and Section 


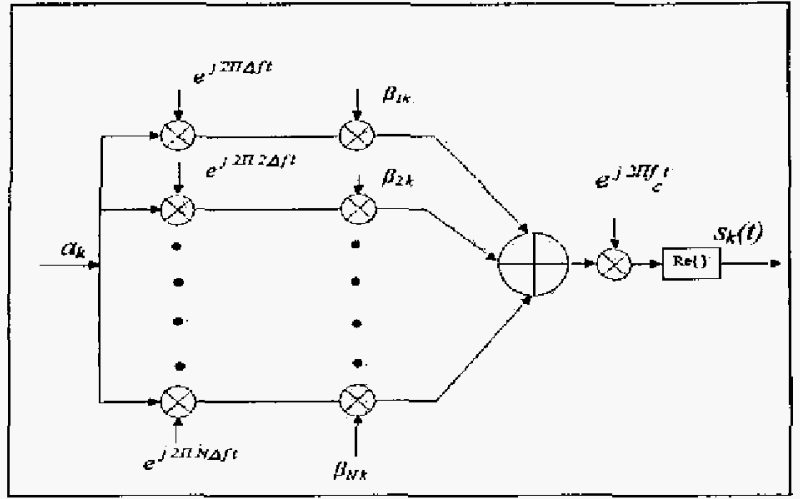

Fig. 1. Conceptual MC-CDMA Transmitter

IV describes serial interference cancellation technique and the different ways of ordering the users. Section $V$ provides the performance results and a brief conclusion is provided in Section VI.

\section{SYSTEM MODEL}

MC-CDMA was first proposed in [10] and is a combination of CDMA and OFDM with the spreading codes applied in frequency domain. CI/MC-CDMA is an MC-CDMA scheme employing complex Carrier Interferometry (C1) spreading codes. The CI code for $k^{\text {th }}$ user corresponds to $\left[\beta_{k}^{0}, \beta_{k}^{1}, \cdots \cdots, \beta_{k}^{N-1}\right]$, where $\beta_{k}^{i}=e^{j i \Delta \theta_{k}}$ [1]. For an orthogonal $\mathrm{Cl}$ code set, it can be easily shown that $\Delta \theta_{k}=\left(\frac{2 \pi}{N}\right) k, k=0,1, \cdots, N-1$. The $C I$ codes of length $N$ have a unique feature that allows CI/MC.CDMA systems to support $N$ Isers orthogonally, and then. as system demand increases, codes can be selected to accommodate up to an additional $N-1$ users pseudoorthogonally. Additionally, there is no restriction on the length $N$ of the $\mathrm{Cl}$ code (i.e., $N \in J$ ) making it more suitable for diverse wireless environments. In contrast. many orthogonal code sets have stringent length restrictions, e.g., where $N$ is limited to $2^{n}$ or $2^{n} \pm 1$ ( $n$ c $I$ ). In this work, we will restrict our analysis to MC-CDMA uplink employing the orthogonal $\mathbf{C l}$ code set.

\section{A. Transmitter Model}

The CI/MC-CDMA transmitter for the $k^{\text {th }}$ user is shown in Fig. 1. The incoming data $a_{k}$ is transmitted over $N$ narrow band subcarriers each multiplied with an element of the $k^{\text {th }}$ user's spreading code. For ease in presentation. BPSK modulation is assumed through out this work, i.e., $a_{k}= \pm 1$. The transmitted signal corresponds to

$$
s_{k}(t)=\sum_{n=-\infty}^{n=+\infty} \sum_{i=0}^{N} a_{k}(n) e^{\left(j 2 \pi f_{i} t+j i \Delta \theta_{k}+\theta_{k}\right)} \cdot P\left(t-n T_{s}\right)
$$

where $f_{i}=f_{c}+i \Delta f$ and $P(t)$ is a rectangular pulse that restricts $s_{k}(t)$ to the symbol duration $\left[0, T_{s}\right]$. As with traditional MC-CDMA and OFDM, $\Delta f$ is selected such that the carrier frequencies $f_{i}, i=$ $0,1, \ldots . N-1$ are orthogonal to each other, i.e., $\Delta f=\frac{1}{T}$. $\Theta_{k}$ denote the random carrier phase of user $k$. In practice, this CVMCCDMA transmitter is implemented using an IFFT followed by a D/A converter similar to that in OFDM. However unlike traditional OFDM, the inpul data stream is subjected to a linear transformation based on the spreading code matrix before entering the IFFT block.

\section{B. Channel Model}

We consider an uplink rayleigh fading channel with correlated envelopes and phases. We assume that every user experiences an independent propagation environment that is modelled as a slowly varying multipath channel. Each path can be modelled in the frequency domain by a zero mean complex gaussian random variable, $\alpha_{i} e^{j \phi_{i}}$, where $\alpha_{i}$ is the lading envelope and $\phi_{i}$ is the random phase introduced by the $i^{\text {th }}$ channel. The fading envelopes are rayleigh distributed with $E\left[\alpha_{i}^{2}\right]=2 \sigma^{2}=1$, where $\mathbf{E}\{\cdot\}$ denotes the expectation. The random phases are uniformly distributed over $[0,2 \pi]$. Multipath propagation in time translates into frequency selectivity in the frequency domain. Frequency selectivity refers to the setectivity over the entire bandwidth of transmission and not over each subcarrier transmission. This is because, $\frac{1}{T_{b}}<<(\Delta f)_{c}<B W$, where $(\Delta f)_{c}$ is the coherence bandwidth and $B W$ is the total bandwidth of the Iransmission. As a result of frequency selectivity over the entire bandwidth. the complex gaussian fades across the sub-carriers are correlated. Assuming both envelope and phase correlation. the corre]ation between any two subcarriers $i$ and $j$ is given by [11]:

$$
\rho_{i j}=\frac{1}{1-j 2 \pi\left(f_{i}-f_{j}\right)\left(\Delta f / \Delta f_{c}\right)}
$$

Furthermore, we also assume that we have an $L$-fold frequency diversity available in the channel, where $L$ is defined as the ratio between the total bandwidth, $B W$, and the coherence bandwidth. $(\Delta f)_{c}$. Given the transmitter and channels models, the next section describes the single user receiver for an MC-CDMA system.

\section{Single User Receiver Model}

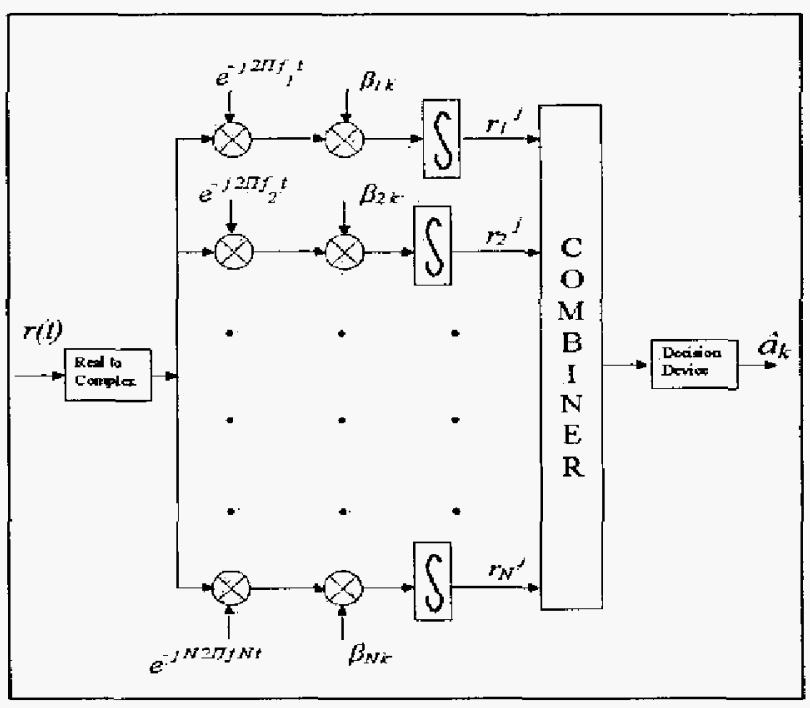

Fig. 2. Conceptual MC-CDMA Receiver

Considering an asynchronous system. the received signal cortesponds to

$$
\begin{aligned}
r(t)= & \sum_{n=-\infty}^{n=+\infty} \sum_{k=1}^{K} \sum_{i=1}^{N} \alpha_{i, k} a_{k}(n) \cos \left(2 \pi f_{i} t+i \Delta \theta_{k}+\varphi_{i, k}\right) \\
& P\left(t-n T_{s}-\tau_{k}\right)+\eta(t)
\end{aligned}
$$

where $\varphi_{i, k}=\Theta_{k}+\phi_{i, k}-\omega_{i} \tau_{k}$, is the net phase shift experienced by user $k . \tau_{k}$, the time delay of user $k$ is uniformly distributed over $\left[0, T_{s}\right) . \alpha_{i, k}$ and $\phi_{i, k}$ are the fading envelope and random phase experienced by user $k$ at the subcarrier $i, \eta(t)$ represents additive white gaussian noise(AWGN). $\alpha_{i, k}, \varphi_{i, k}$, and $\tau_{k}$ are assumed to be independent and identically distributed (iid) for different $k$. The single user receiver for user $j$ is shown in Fig. 2. We consider a correlation receiver with perfect channel estimation. The desired user $j$ is assumed to be the reference user and the delay $\tau_{j}$ is set to zero. 
The received signal is first projected onto $N$ orthogonal carriers and is then despread using $j^{\text {th }}$ users $\mathrm{CI}$ code resulting in $\underline{r}_{-}^{j}=\left(r_{0}^{j}, r_{1}^{j}, r_{2}^{j}, \cdots\right.$ $\cdots r_{N-1}^{j}$ ), whete $r_{p}^{j}$ corresponds to

$$
\begin{aligned}
r_{i}^{j}= & \alpha_{i, j} a_{j}(n) \\
+ & \sum_{k=1, k \neq j}^{k}\left(\alpha_{i, k}\left(a_{k(-1) \tau_{k}}+a_{k(0)}\left(T_{s}-\tau_{k}\right)\right)\right. \\
& =\exp \left(i\left(\Delta \theta_{k}-\Delta \theta_{j}\right)+\varphi_{i, k}-\hat{\varphi}_{i, j}\right)+ \\
& \sum_{p=1, p \neq i}^{N} \alpha_{p, k} \frac{a_{k(-1)}-a_{k(0)}}{j 2 \pi \Delta f t} \exp \left(j 2 \pi \Delta f \tau_{k}(i-p)\right. \\
& \left.+\left(i \Delta \theta_{k}-p \Delta \theta_{j}\right)+\varphi_{i, k}-\hat{\varphi}_{p, j}\right) \\
+ & \sum_{p=1, p \neq i}^{N} \eta_{i} ;
\end{aligned}
$$

Where $\eta_{i}$ is a Gaussian tandom variable with mean 0 and variance $N_{0} / 2$. Exact phase and frequency synchronization for the desired user is assumed while writing equation (4), i.e.. $\hat{\psi}_{i, j}=\varphi_{i, j}$. Now, a suitable combining strategy is used to create a decision variable variable $D^{j}$, which then enters a decision device that outputs $\hat{a}_{j}$. The decision variable $D^{j}$ of the $0^{t h}$ data bit of user $i$ is given by

$$
\begin{aligned}
D^{j} & =\sum_{i=1}^{N} \omega_{i, j} r_{i}^{j} \\
& =S_{j}+\eta_{j}+M A I_{j}
\end{aligned}
$$

where $S_{j}, \eta_{j}, M A I_{j}$ are the desired signal term, the AWGN noise term and multiple access interference (MAI) respectively and $\omega_{p, j}$ is the combining parameter. In this work, minimum mean square enror combining (MMSEC) is employed as it is shown to provide the best performance in a frequency selective fading channel [12].

$$
\omega_{p, j}=\alpha_{p, j} /\left(\operatorname{var}\left(a_{k}\right) A_{p, j}+\frac{N o}{2}\right)
$$

where $A_{p, j}=\sum_{k=1}^{N} \alpha_{i, k}^{2} \cos ^{2}\left(i \Delta \theta_{k}-p \Delta \theta_{j}+\varphi_{k, p}-\varphi_{j, p}\right)\left(r_{k}^{2}+\right.$ $\left.\left(T_{s}-\tau_{k}\right)^{2}\right)$, $\operatorname{var}\left(a_{k}\right)=1$. The desired signal $S_{j}$ corresponds to

$$
S_{j}=a_{j}(0) \sum_{p=1}^{N} \alpha_{p j} \omega_{p, j}
$$

The noise term $\eta_{p}$ is a gaussian randon variable with mean zero and variance $N o / 2 \sum_{p=1}^{N} \alpha_{j, i}^{2}$. The $M A I_{j}$ can be written as

$$
M A I_{j}=\sum_{k=1, k \neq j}^{K}\left(I_{j, k}+J_{j, k}\right)
$$

where $I_{j, k}$ and $J_{j, k}$ represent MAI from the same subcarriers $(i=p)$ and other subcarriers $(i \neq p)$, respectively. They are given by

$$
\begin{aligned}
I_{i, k}= & \sum_{p=1}^{N} \omega_{p, k} \alpha_{p_{r} k}\left[a_{k(-1)} \tau_{k}+a_{k(0)}\left(1-\tau_{k}\right)\right. \\
& \left.\cos \left(\varphi_{k, p}-\varphi_{j, p}+p\left(\Delta \theta_{k}-\Delta \theta_{j}\right)\right)\right]
\end{aligned}
$$

and

$$
\begin{aligned}
J_{i, k}= & \sum_{i=1}^{N} \sum_{p=1, p \neq i}^{N} \alpha_{p, k} \omega_{p, k}\left(a_{k(0)}-a_{k(-1)}\right) \\
& (10) \\
& \left(\operatorname { s i n } \left[\left(\omega_{p}-\omega_{i}\right) \tau_{k}\right.\right. \\
& \frac{\left.\left.+\left(\varphi_{k, i}-\varphi_{j, p}\right)\right]-\sin \left(\varphi_{k, i}-\varphi_{j, p}\right)\right)}{(2 \pi \Delta f(i-p))}
\end{aligned}
$$

Thus, the outputs of all the single user detectors of all users generate a decision vector $\underline{D}=\left[D^{1} D^{2} \ldots \cdots D^{K}\right]$ which is used to obtain the initial estimates of the data $\underline{\hat{a}}=\left(\hat{a}_{1}, \hat{a}_{2} \cdots \hat{a}_{\kappa}\right)$. These initial estimates are then used to evaluate the MAI experienced by each user in the interference cancellation techniques discussed in the next section.

\section{BLOCK-PIC}

In conventional parallel interference cancellation. the MAJ experienced by the desired user due to every other user accessing the channel is cancelled at once [13]. In this work, we assume that the receiver has perfect knowledge of the channel state of all users. The initial estimates from the single user detectors described in Section II, are used to regenerate the signals of the interfering users at the receiver.The regenerated signal of user $k$ using the estimated data $\hat{a}_{k}$ corresponds to $\hat{r_{k}}(t)=\hat{a_{k}} \sum_{i=1}^{N} \omega_{k i} \alpha_{k i} \beta_{k i} e^{\left(\omega_{k i}+2 \pi\{i \Delta f) t\right)}$. The regenerated signals of all interfering users are removed from the desired user and the interference free signal for user $j$ is given by

$$
r^{\prime}(t)=r(t)-\sum_{k=1, k \neq j}^{K} \hat{r_{k}}(t)
$$

This $r^{\prime}(t)$ is once again fed into the single-user detector described in Section II for a better estimate of the data. After projecting $r^{\prime}(t)$ onto the orthonormal basis and despreading, the combining technique is employed. However the MMSEC weights are updated in order to account for the interference cancellation [14]. Specifically, the updated weights correspond to

$$
\omega_{i j}=\frac{\alpha_{i j}}{\operatorname{var}\left(a_{k}-\hat{a_{k}}\right) B_{p, j}+\frac{N_{o}}{2}}
$$

where $B_{p, j}=\sum_{k=1}^{N} \alpha_{i k}^{2}\left(\beta_{i k} \beta_{i j} \cos ^{2}\left(\varphi_{k i}-\varphi_{j i}\right)^{2}\right.$. The weights in equation lil can be calculated in two ways: (1) if the estimates are assumed to be correct then $\operatorname{var}\left(a_{k}-\hat{a}_{k}\right)=\operatorname{var}\left(a_{k}-a_{k}\right)=0 \forall$ $k \neq j:$ (2) if there is no prior knowledge about the reliability of the estimates, then $\operatorname{var}\left(a_{k}-\hat{a}_{k}\right)=2 \forall k \neq j$ ( since $\left(a_{k}-\hat{a}_{k}\right)$ may be modeled as discrete random variable which can take values 2, 0 and -2 ). It can be observed that conventional PIC provides

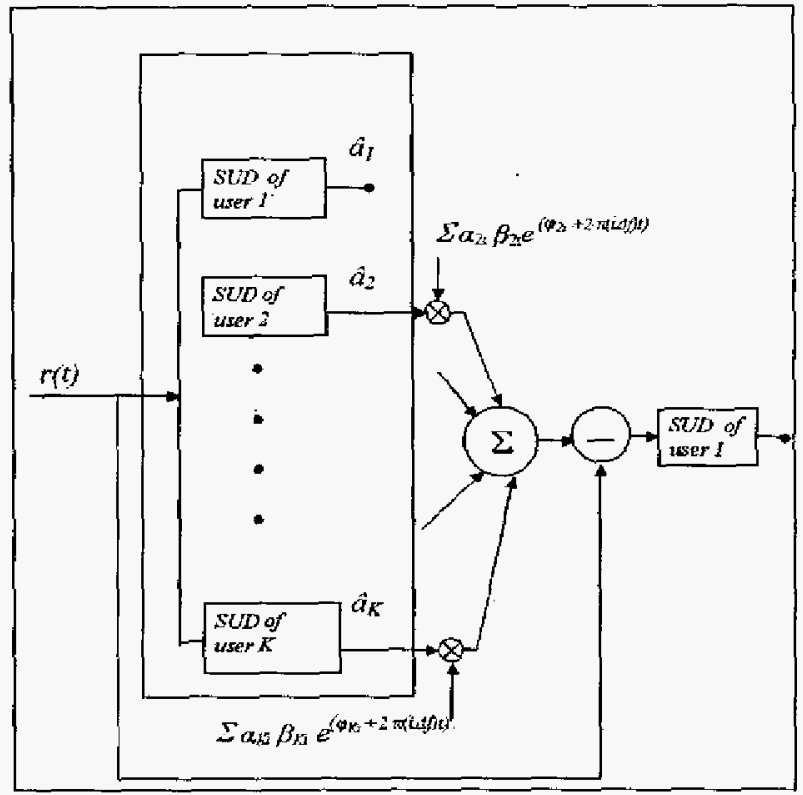

Fig, 3. PIC receiver for user 1

better performance when the weights are calculated by the second method as we are subtracting the interference due to every other user 


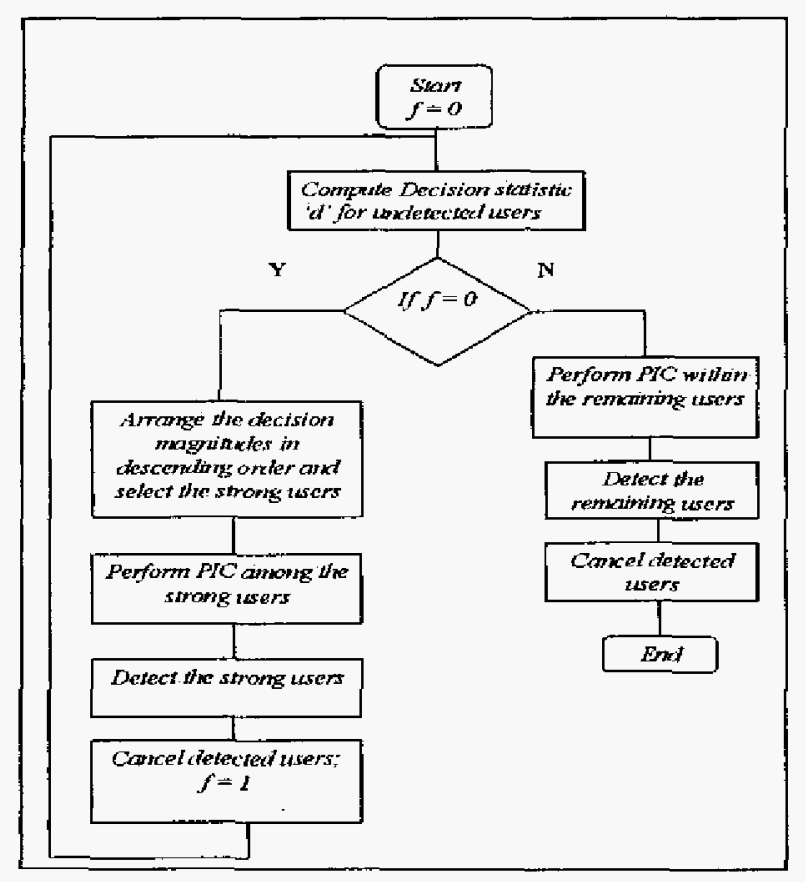

Fig. 4. Block-PIC Algorithm

accessing the channel irrespective of their strengths. On the other hand Block-PIC provides better performance when the weights are calculated assuming perfect detection as we are detecting the strong users first. The algorithm for Block-PIC is shown in figure III. In this method the users are ranked according to the magnitudes of the decision statistics and are arranged in descending order. The first half of the users are considered as strong users' ( Block-1) and second half as weak users ( Block-2). Now PIC is performed within Block-1 and the strong users" data is estimated. Using this updated data the interference due to these users is removed from Block 2. Then. PIC is employed within Block-2 and the weak users' data is estimated. The performance can be further improved by increasing the number of blocks but at the cost of increase in complexity.

\section{DYNamically ORDERED SIC}

Serial interference cancellation is a multiuser detection technique in which users are deiected sequentially based on the reliability of the signals [15]. Once again, we assume that the receiver has perfect knowledge about the channel state of all users. The users are arranged in descending order based on the magnitude of their decision statistics. The user with maximum magnitude is detected first and the corresponding signal at the receiver is regenerated. The regenerated signal of user $k$ using the estimated data $\hat{a}_{k}$ corresponds to $r_{k}(t)=\hat{a_{k}} \sum_{i=1}^{N} \alpha_{k i} \beta_{k i} e^{\left(\varphi_{k i}+2 \pi(i \Delta f) t\right)}$ The regenerated signal is cancelled from the teceived signal. The received signal after $r$ cancelations corresponds to

$$
r^{\prime}(t)=r(t)-\sum_{k=1}^{r} I_{k}(t)
$$

This $r^{\prime}(t)$ is once again fed into the single-user detector described in Section II. After projecting $r^{\prime}(t)$ onto the orthonormal basis and despreading, the MMSEC combining is employed. Now the Minimum Mean Square Error Combining is employed to this interference free signal, in order to take the interference cancellation into effect[14].

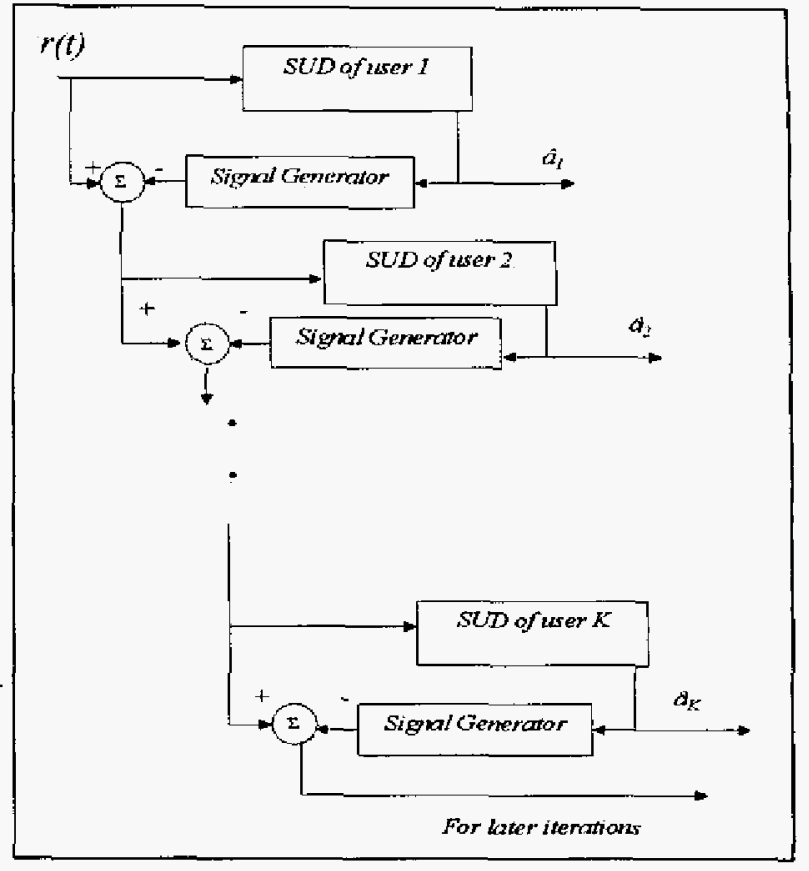

Fig. 5. SiC Block Diagram

The updated weights corresponds to

$\omega_{i j}=\frac{\alpha_{i j}}{\operatorname{var}\left(a_{k}\right) \sum_{k=r+1}^{K} \alpha_{i k}^{2} \cos ^{2}\left(i \Delta \theta_{i}-i \Delta \theta j+\phi_{k i}-\phi j i\right)+\frac{N o}{2}}$

Once again. the weights can be calculated in two ways: (1) Assuming that the estimated data is correct and (2)Assuming that there is no prior knowledge about reliability on the estimates. In this scheme the order is recomputed after each interference cancellation. The algorithm for this scheme is described below: for $m=1: M\{$

$\mathbf{L}=$ set of users indexed from 1 to $K$ while $L \neq \emptyset$ do (

1. $Q_{j}^{\text {nt }}(t)=r(t)+I_{j}^{m}(t)$ for all $j \epsilon L$

2. Determine $D_{j}^{m}$ from $Q_{j}^{m}(t)$ for all $j \epsilon L$

3. Find $i$ such that $\left|D_{i}^{m 2}\right|$ is $\max$

4. $\hat{a}_{i}^{m}=\operatorname{sgn}\left(D_{i}^{\text {tn }}\right)$

5. Compute the regenerated signal for user i. $I_{i}^{m}(t)$

6. $r(t)=r(t)+I_{i}^{m-1}(t)-I_{i}^{m}(t)$

7. Remove $i$ from set $L$

\}

\}

In the DO-II algorithm. $L$ is the set of undetected users in no specific order. As before, $I_{j}^{O}(t)$ is set to $0 \forall j \in 0,1, \cdots K$. In the tirst cancellation stage, $Q_{j}^{1}(t)=r(t)$ and is used to compute the decision statistic for all users. The user with maximum decision statistic magnitude is detected first and its contribution to $r(t)$ is cancelled. This user is removed from the set $L$ and the process is repeated until all users are detected. In later fletations, the $r(t)$ that enters the cancellation loop consists of channel noise and residual MAI (similar to fixed ordering). At each cancellation stage the residual noise signal is updated based on the new estimate of the data symbol (see step 6).

\section{Performance Results}

We consider an asynchronous $\mathrm{Cl} / \mathrm{MC}$-CDMA uplink with each users" channel modelled as slow frequency selective fading channel 
with four-fold diversity (i.e.. $L=4$ ). We investigate the BER performance with different spreading codes. MRC combining is employed. The results are presented in Table V. We observe from the table that the BER performance of asynchronous MC-CDMA system is similar with all spreading codes.

\begin{tabular}{llllll}
\hline Eb/N0 & $\begin{array}{l}\text { Users K=8 } \\
\text { OdB }\end{array}$ & $10 \mathrm{~dB}$ & $20 \mathrm{~dB}$ & $30 \mathrm{~dB}$ & $40 \mathrm{~dB}$ \\
\hline Walsh & 0.1569 & 0.0898 & 0.0780 & 0.0767 & 0.0775 \\
Gold(N=7) & 0.1754 & 0.1064 & 0.0970 & 0.0961 & 0.0875 \\
Random & 0.1657 & 0.0883 & 0.0831 & 0.0806 & 0.0802 \\
Cl & 0.1612 & 0.0869 & 0.0771 & 0.0762 & 0.0769 \\
\hline
\end{tabular}

BER(PF)AGAAINST $E_{b} / N_{0}$ FOR ASYNCHRONOUS MC-CDMA WITH DIFFERENT SPREADING SEQUFNCES, MRC COMUINING $(N=8$, EXCEPT FOR THE CASF OF GOLD SEOUENCES)

We consider a $50 \%$ loaded system with a processing gain of $N=$ 32. MMSEC is employed. Figure 6 compares the performance of conventional PIC and Block-PIC schemes. It is evident that BlockPIC significantly outperforms conventional PIC and provides a gain of $8 \mathrm{~dB}$ at a BER of $10^{-2}$. It is also observed that with conventional PIC, a residual BER (error floor) of approximately $\cdot 10^{-2}$ exists at higher SNR. This error floor is app $4 \cdot 10^{-3}$ with Block-PIC.

Figure 7 compares the performance of fixed and dynamic ordering in an asynchronous MC-CDMA system employing SIC. A gain of $8 \mathrm{~dB}$ is achieved with dynamic ordering relative to fixed ordering at a BER of $2 \cdot 10^{-3}$. It is also observed that dynamic ordering scheme is $4 \mathrm{~dB}$ off from single user performance at a BER of $10^{-4}$.

\section{CONCLUSIONS}

In this paper. we analyze the performance of asynchronous CI/MC. CDMA uplink with new variants of interference cancellation. We demonstrate that Block-PIC outperforms conventional PIC with a gain of $10 \mathrm{~dB}$ at a BER of $10^{-2}$. We also analyze effect of order of cancelling on BER performance with serial interference cancellation receiver. We observe significant improvement in performance $(8 \mathrm{~dB}$ at a BER of $2 \cdot 10^{-3}$ ) with dynamic ordering relative to fixed ordering.

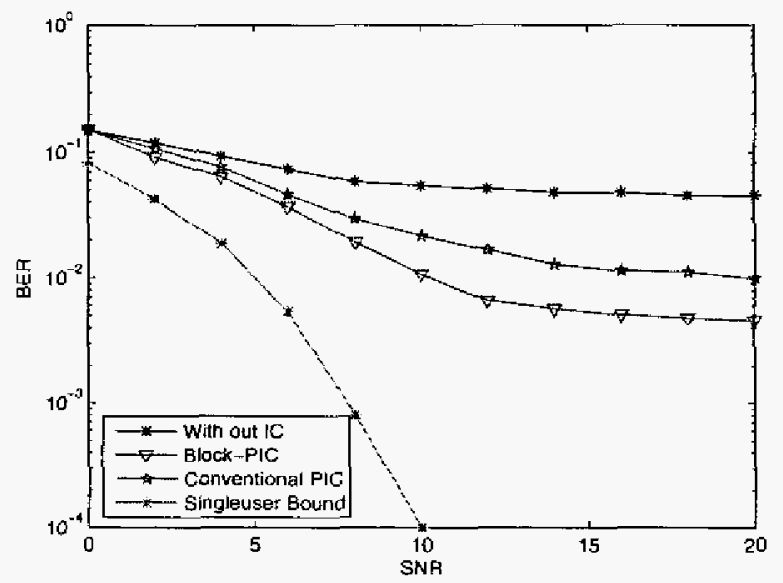

Fig. 6. Performance comparison of asynchronous system employing BlockPIC and conventional PIC

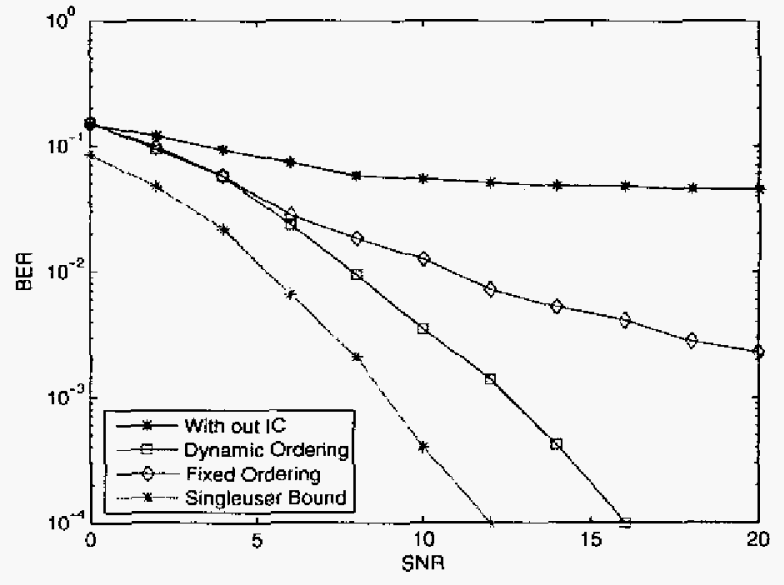

Fig. 7. Performance comparison of asynchronous system employing Fixed Ordering and Dynamic Ordering

\section{REFERENCES}

[t] B. Natarajan. C. R. Nassar. S. Shattil, M. Michelini, and Z, Wu. "High-performance inc-cdma via carrier interferometry codes," IEEE Transactions on Vehictlar Technology , vol. 50, pp. 1344 - 1353, 2001.

[2] S. Verctu, "Minimum probability of error for asynchronous gaussian multiple-access channels," IEEE Trunsactions on Information Theory. vol. 32. pp. 85-96, 1986.

[3] R.Lupas and S.Verdu, "Linear multiuser detectors for syrnchronous code-division multilpe-access channels," IEEE Transactions on Inform. Theory, vol. 35, pp. 123-136, 1989.

[4] Y. Li and R.Steele, "Serial interference cancellation method for CDMA," Electronics Letters, vol. 30, pp. 1581-1583, I994.

[5] D. Hong. Y. You, and C. Kang. "Pipelined successive interference cancellation scheme for a DS/CDMA system.," IEEE Commun letter. in press. vol. 3. pp. 2045-2048. May 1997.

[6] T. Kim, D. Hong, C. Kang, and D. Hong, "Adaptive pipelined suecessive interference cancellation scheme for a DS/CDMA system." IEEE 49th Vehicular Tecthology Conff. Phoenix.AZ, vol. 3. pp. 740-743. 2000.

[7] V. Thippavajjula and B. Natarajan, "Performance of serial interference cancellation receivers in synchronous carrier interferometry/MC-CDMA uplink," in Proceedings of the Intemational Conference on Wireless Networks, Accepted for Publication, June 2004.

[8] D. Divsalar and M. Simon, "A new approach to parallel interference cancellation for CDMA," Global Telecommunications Conference, vol. 3. pp. 1452-1457, 1996.

[9] V.Thippavajjula and B.Natarajan. "Parallel Interference Cancellation Techniques for Synchronous Carrier Interferometry/MC-CDMA Uplink.," IEEE Transactions on Vehicular Tecinology, pp. 69-78, Sept,2004

[10] N.Yee. J.P.Linnanz, and G.Fettweis, "BER of multi-cartier CDMA in indoor wireless networks," IEEE Transactions on Communications, vol. 1. pp. 2817-2821, 1993.

[11] W. C. Jakes. Microwave Mobile Communications. New York: IEEE Press, 1974.

[12] X. Cai and A. N.Akansu, "Multicarrier CDMA systems with transmit diversity." IEEE Tunsactions on Vehicular Techology., vol. 2. pp. 658661.2000.

[13] R.Fantacci, S.Morosi, and F.Panchetti, "One-Shot Multi-User Cancellation Receiver for WIRELESS CDMA. Communication Systems.," IEEE Transactions on Vehicular Technology. pp. 1134-1 138, 1999.

[14] L. Xiao and Q. Liang, "The study of parallel interference weighted canceller multiuser detection." IEEE VTS 50ti Vehicular Technology Conference, vol. 5, pp. $3009-3013,1999$.

[15] P.Patel and J.Holtzman, "Analysis of a Simple Successive Interference Cancellation Scheme in a DS/CDMA System.." IEEE trans. Communicutions. pp. 796-807, 1994. 